\title{
Technologies Serving Concrete for Economy, Durability and Sustainability
}

\author{
Yahia Alhassani \\ Yahia.alhassani@parsons.com \\ Parsons International LLD, Doha, Qatar
}

\begin{abstract}
Concrete comes as the second most used material after water in the world, but it is the first used construction material especially in Middle East. World population is increasing while resources are decreasing. Therefore, construction industry needs to find a way to produce more by consuming less. Currently, concrete production results in significant carbon dioxide emissions creating an environmental hazard. On the other hand, usable sand for concrete in many countries is short in supply. Hence, science and technology need to be used at the earnest to prevent unrecoverable environmental damage associated with elevated costs. This paper describes a recently developed technology that converts desert sand into construction sand and aggregates when combined with another technology of dual mixing scheme capable of providing up to $40 \%$ of cement savings in concrete production; remarkable results are achieved. Those revolutionary, economical and environment friendly technologies are now available. We will introduce the outlines of the said technologies and then we shall present the possible applications of these technologies for efficient, sustainable and durable construction especially for the area.
\end{abstract}

\section{INTRODUCTION}

Concrete exhibits many advantages for the construction industry such as excellent resistance to water and fire, is generally a durable material, flexible for molding and shaping, usually made of local ingredients and relatively inexpensive. The idea of mixing water, binder and stones to formulate such plastic material with ability to be casted in predefined shape is very old and could go back to 20 centuries. Such composite called concrete was developed with time and according to needs along with the available technologies. Of course, the invention of Portland Cement and after that the admixtures are considered revolutionary in concrete industry. These developments enabled concrete to be a widely recognized and highly standardized construction building material, used several times more than steel or timber in many countries. Precast technologies helped much to have concrete products with higher quality and quick erection. Despite all these developments, one critical feature of concrete production remained unchanged over centuries; the mixing process. Mixing such composite of multi-scales (few micrometers to several millimeters) in conventional way presents a limitation to optimize concrete properties as still not complying to produce fully homogenous mixture. Dual Mixing is our first technology to speak about in this paper.

Other challenge or obstacle is to have enough resources for construction and concrete in order to have the required housing and infrastructure and follow the boom in such developments is finding enough suitable aggregates. This problem is faced in most of the countries that are laying in desert zones. The solution of importing the aggregates from other countries usually imposes additional challenges and issues with logistics, extra 
cost, less control on sources and sometimes internal relationship restrictions in addition to environmental adversity (Dredging Activities if Applied).

As we know, the world's population is increasing, and most of that increase will be in our area (Asia, Middle East and Africa). So, we need to find a way to produce more in order to satisfy the needs and at the same time should be economical and environment friendly/sustainable. Since the desert sand is widely available, and we usually remove it from construction sites. New technology was developed to convert desert sand into aggregates in palletization process with the help of Dual Mixing technology as mentioned before. This technology will be presented in the second points in our paper.

\section{MATERIALS AND METHODS}

\section{2.i Dual Mixing}

The idea of Dual Mixing came over by questioning the way of conventional concrete mixing. By mixing water, cement and aggregates with a low speed mixer (tens rpm) for several minutes will not enable all cement particles to get in touch with needed water, hence, more water and cements are used to achieve the target slump and strength. It is well known that the water cement ratio needed for the hydration process is in the order of 0.15 , whereas the rest of the water is needed for plastic consistency, i.e. slump for workability. In other words, to increase the probability of having cement particles meet with water, higher dosage of cement is usually used requiring higher water consumption. In order to devise an optimum concrete, the function of water is split into two by changing the mixing process to a dual mixing strategy patented by Multicon $\mathrm{GmbH}$. The idea of Dual Mixing consists of mixing the slurry components (Water, binder and admixture) in very high-speed mixer ranging $1500 \mathrm{rpm}$ ) and added to the aggregates (coarse $\&$ fine). To show the difference between the two ways of producing concrete and achievements we will prepare a mix presenting each of them. The first mix is prepared with water, cement, cement replacing inert fines and admixtures by using an advanced high-speed mixer at $1500 \mathrm{rpm}$ at about 20 seconds. The water binder ratio is kept at about $30 \%$ and cement replacing inert materials (for example limestone powder) with a maximum size of 100 micrometer is used to reduce cement consumption up to $40 \%$. The use of high-speed mixing improves liquefaction of concrete, disperses the cement particles effectively, further breaks the particles by abrasion and allows obtaining a highly homogenized mix with an optimum $\mathrm{w} / \mathrm{c}$ ratio. The second mix is conducted like the conventional concrete mixing with the slurry from the first mix, aggregates and additional water, if needed, for the target slump. The flowchart of the dual mixing process is summarized in Figure 1. a and 1.b. An example mix design and obtained savings are presented in Figure 1.c. It can be observed that the cement saving, and by consequence carbon dioxide emission reduction is (about $30 \%$ ) with a cost saving of about $15 \%$. 


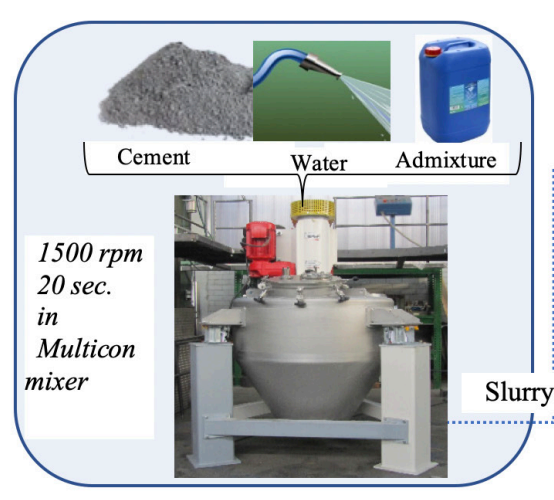

a) Stage 1- High Speed Mixing.

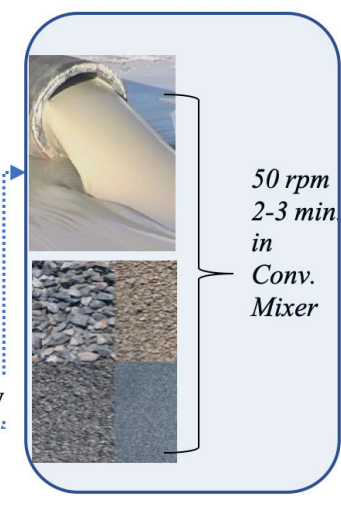

b) Stage 2- Conv. Mixing

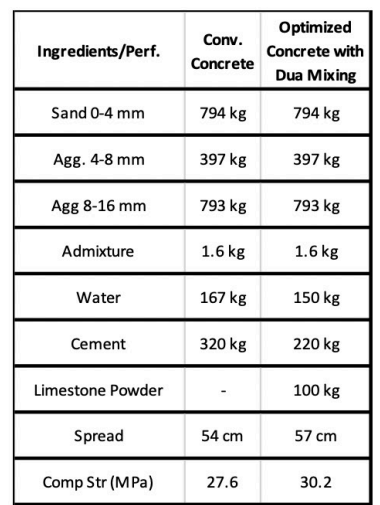

c) Mixes (typical improvement)

Figure 1: Dual Mixing Methodology and Benefits

The dual mixing technology does not only provide economical and sustainable concrete but also allows the realization of lightweight concrete in an efficient manner. The researchers of Multicon invented perlite lightweight concrete produced by using perlite, cement, water and admixtures mixed with the advanced high-speed mixing technology. The technology allows producing concrete with a specific weight of about $1500 \mathrm{~kg} / \mathrm{m}^{3}$ and a strength of $30 \mathrm{MPa}$ (Figure 2.a). Such a concrete can reduce gravity and earthquake forces thereby providing economical and more efficient transportation of precast elements. In the case of using aluminum powder as the expansion agent, we may produce aerated concrete with a specific weight of $700 \mathrm{~kg} / \mathrm{m}^{3}$ and strength of about $6 \mathrm{MPa}$. In this way non-structural lightweight concrete block can be produced without needing any expensive autoclaving process (Figure 2.b).

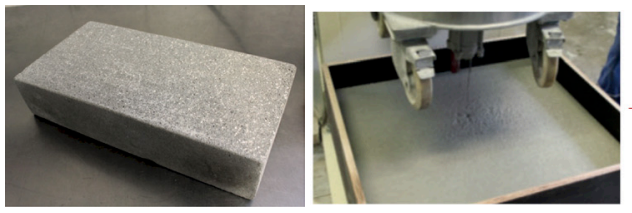

a) Lightweight concrete

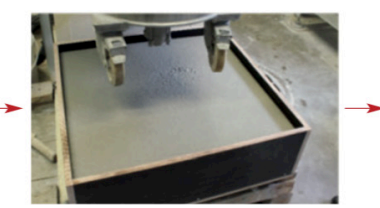

b) Lightweight aerated concrete

Figure 2: Lightweight concrete with high speed mixing

The other good advantage of Dual Mixing technology is helping to produce Self Compacting Concrete (SCC) easily and with less cost.

\subsection{Sand Aggregates}

Despite the wide availability of concrete ingredients, some countries, especially those having large deserts, lack quality aggregate necessary for concrete production. The transportation of aggregates is almost never a feasible option; hence sand is usually extracted from river or sea basins with consequential irreversible environmental damage. Multicon $\mathrm{GmbH}$ recently developed a procedure to address the needs of the society. With this patented technology, the abundant useless desert sands can be converted into usable construction sands or aggregates such as sand and gravel. With this technology, 
the fine or desert sand is first pulverized with grinding mills into finer particles (Figure 3). This sand powder is then granulated on pelletizing plates with the addition of the slurry similarly obtained in the first stage of the Dual Mixing Technology. More than $90 \%$ of grains have a sphericity above 0.9 (GmbH 2018), and sizes vary between 0.1 to $16 \mathrm{~mm}$ with a compressive strength of 10 to $100 \mathrm{MPa}$ made with a 5 to $15 \%$ cement consumption. In this way, man-made natural aggregates are prepared from dessert sand and slurry from the high-speed mixing process. Properties of pellets concrete were investigated by an accredited institution (IAB, 2019).

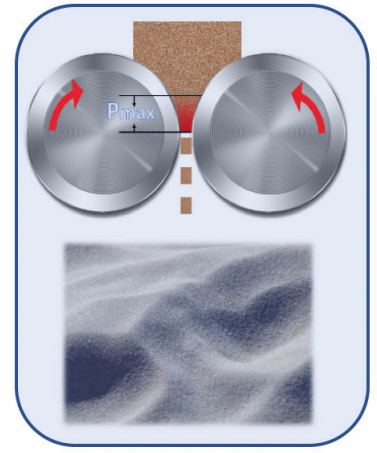

a) Pulverization

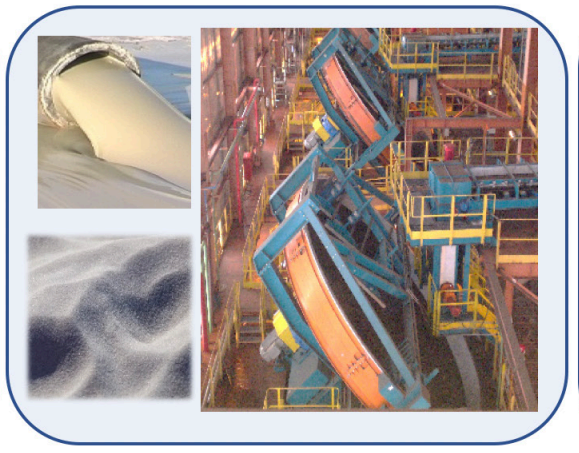

b) Pelletization

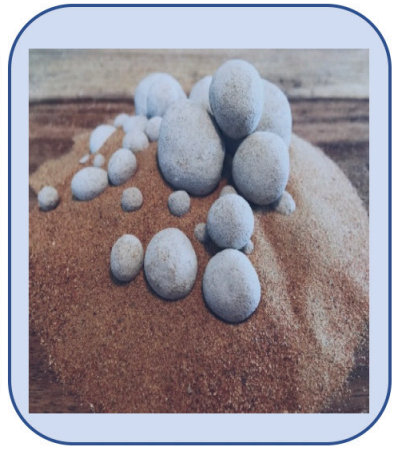

c) Sand-aggregates

Figure 3: Production of Sand Aggregates

Figure 4 shows the Sand aggregates (pellets), and cross section of concert made of. The spherical shape is very helpful for flowability and pumpability of concrete.

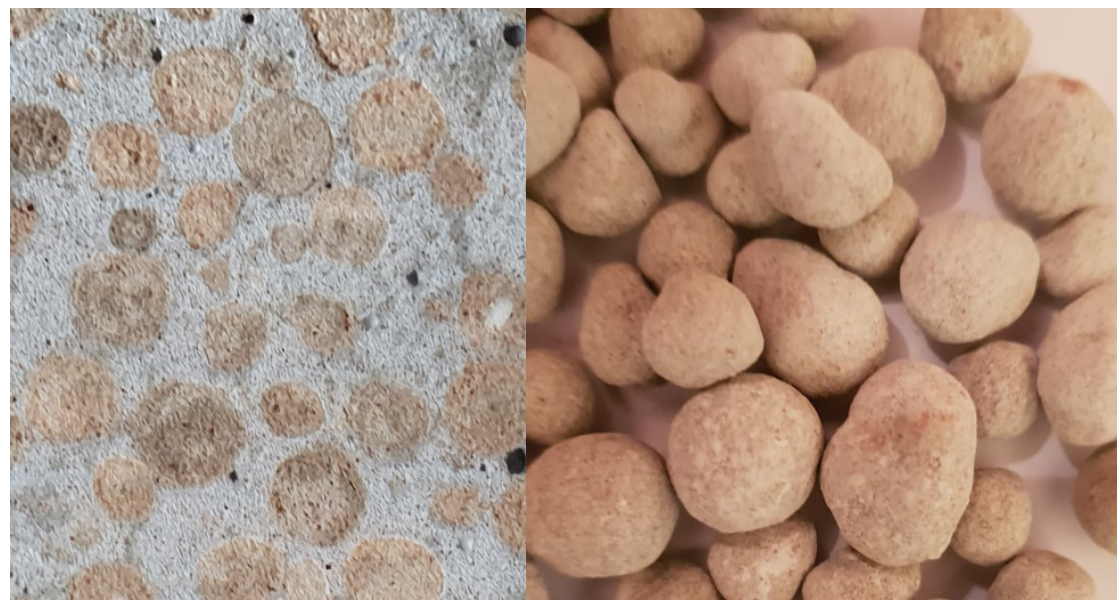

a) Pellets

b) Concrete Cross section

Figure 4: Sand aggregates and Concrete.

\section{CONCLUSION}

The conventional way of mixing concrete is a serious barrier in improving the properties of concrete, providing economy and increasing sustainability. Thanks to the 
invention of the dual mixing strategy with the advanced high-speed mixer of Multicon $\mathrm{GmbH}$, new frontiers are now wide open for better concrete. The future vision of the technology places the high-speed mixer at the heart of concrete production. The slurry produced from the high-speed mixer provides the binder to produce pelletized aggregates utilizing abundant desert sand while preserving river basins and environmental damage. Afterwards, pelletized aggregates and slurry from the first stage high speed mixing are conventionally mixed to produce cement optimized environment friendly, sustainable concrete. The reinvention presented herein not only reduces cement consumption in concrete production, reduces emissions and protects the environment with the utilization of desert sand but also increases sustainability and brings a new vision for the future of concrete.

\section{REFERENCES}

DIN EN 12620 (Feb. 2019). IAB - Institut für Angewandte Bauforschung Weimar gemeinnützige $\mathrm{GmbH}$.

Pelletizing of desert sand (October 2018). Technical Report, HAVER ENGINEERING GmbH.

Prüfbericht PB 1916, Untersuchung von Sand-Zement-Agglomeraten auf Eignung als Gesteinskörnung für Beton nach. 